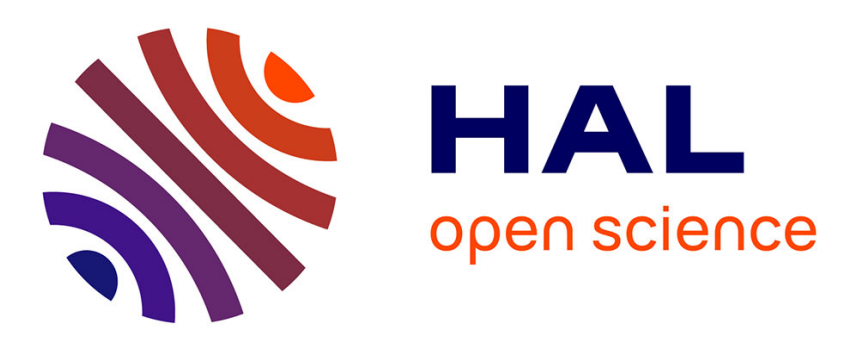

\title{
Sharp estimates of bounded solutions to some second order forced dissipative equations
}

\author{
Alain Haraux
}

\section{To cite this version:}

Alain Haraux. Sharp estimates of bounded solutions to some second order forced dissipative equations. 2006. hal-00113444

\author{
HAL Id: hal-00113444 \\ https://hal.science/hal-00113444 \\ Preprint submitted on 13 Nov 2006
}

HAL is a multi-disciplinary open access archive for the deposit and dissemination of scientific research documents, whether they are published or not. The documents may come from teaching and research institutions in France or abroad, or from public or private research centers.
L'archive ouverte pluridisciplinaire HAL, est destinée au dépôt et à la diffusion de documents scientifiques de niveau recherche, publiés ou non, émanant des établissements d'enseignement et de recherche français ou étrangers, des laboratoires publics ou privés. 


\title{
Sharp estimates of bounded solutions to some second order forced dissipative equations
}

\author{
Alain Haraux
}

Résumé. A l'aide d'inégalités différentielles, on établit une estimation proche de l'optimalité pour la norme dans $L^{\infty}(\mathbb{R}, V)$ de l'unique solution bornée de $u^{\prime \prime}+$ $c u^{\prime}+A u=f(t)$ lorsque $A=A^{*} \geq \lambda I$ est un opérateur borné ou non sur un espace de Hilbert réel $H, V=D\left(A^{1 / 2}\right)$ et $\lambda, c$ sont des constantes positives, tandis que $f \in L^{\infty}(\mathbb{R}, H)$.

Abstract. By using differential inequalities, a close-to-optimal $L^{\infty}(\mathbb{R}, V)$ bound of the unique bounded solution of $u^{\prime \prime}+c u^{\prime}+A u=f(t)$ is obtained whenever $A=A^{*} \geq$ $\lambda I$ is a bounded or unbounded linear operator on a real Hilbert space $H, V=D\left(A^{1 / 2}\right)$ and $\lambda, c$ are positive constants, while $f \in L^{\infty}(\mathbb{R}, H)$.

Keywords: second order equation, bounded solution

AMS classification numbers: 34C15,34C25, 34C27, 34D05, 34D030 


\section{Introduction}

Let $H$ be a real Hilbert space. In the sequel we denote by $(u, v)$ the inner product of two vectors $u, v$ in $H$ and by $|u|$ the H-norm of $u$. Let $A: D(A) \rightarrow H$ a possibly unbounded self-adjoint linear operator such that

$$
\exists \lambda>0, \forall u \in D(A), \quad(A u, u) \geq \lambda|u|^{2}
$$

We consider the largest possible number satisfying the above inequality, in other terms

$$
\lambda_{1}=\inf _{u \in D(A),|u|=1}(A u, u)
$$

We also introduce

$$
V=D\left(A^{1 / 2}\right)
$$

endowed with the norm given by

$$
\forall u \in V, \quad\|u\|^{2}=\left|A^{1 / 2} u\right|^{2}
$$

We recall that

$$
\forall u \in D(A), \quad\left|A^{1 / 2} u\right|^{2}=(A u, u)
$$

It is clear that the norm just defined on $V$ is equivalent to the graph norm of $A^{1 / 2}$ as a consequence of our coerciveness assumption on $A$.

Given $f \in L^{\infty}(\mathbb{R}, H)$ the second order evolution equation

$$
u^{\prime \prime}+c u^{\prime}+A u=f(t)
$$

is well-known to have a unique bounded solution

$$
u \in C_{b}(\mathbb{R}, V) \cap C_{b}^{1}(\mathbb{R}, H)
$$

Indeed, putting (1) in the equivalent form

$$
u^{\prime}=v ; \quad v^{\prime}+c v+A u=f
$$

and introducing the contraction semi-group $S(t)$ generated on $V \times H$ by the system

$$
u^{\prime}-v=v^{\prime}+c v+A u=0
$$


since $S$ is exponentially damped on $V \times H$, we have (cf.e.g. [5])

$$
\forall t \in \mathbb{R}, \quad\left[u(t), u^{\prime}(t)\right]=\int_{0}^{\infty} S(\tau)[0, f(t-\tau)] d \tau=\int_{-\infty}^{t} S(t-s)[0, f(s)] d s
$$

Assuming

$$
\|S(t)\| \leq M e^{-\delta t}
$$

we also get the estimate

$$
\forall t \in \mathbb{R}, \quad\|u(t)\| \leq\left\{\|u(t)\|^{2}+\left|u^{\prime}(t)\right|^{2}\right\}^{1 / 2} \leq \frac{M}{\delta}\|f\|_{L^{\infty}(R, H)}
$$

However, in applications to infinite dimensional systems such as the wave equation, the standard method relying on the above formulas does not seem to give the best possible estimate of the energy because the choice of $\delta$ and $M$ is not easy to optimize, especially in presence of double eigenvalues or more generally close enough eigenvalues. The object of this paper is to develop specific methods to obtain a good estimate on the $L^{\infty}$ norm of $u$ with values in $V$. Not too surprisingly the results which we obtain are natural extensions of optimal estimates for second order ODE which were devised in [7] for the special case of Duffing's equation. Therefore these result are in the direct line of the tradition initiated by Cartwright and Littlewood [2] and continued by W.S. Loud [8]. Duffing's equation have been studied a lot both theoretically and numerically, cf. e.g. [3, 4, 10]. Infinite dimensional variants of Duffing's equation arise naturally in continuum mechanics. In this framework the behavior of the general solution becomes very complicated but it is still useful to derive sharp estimates for bounded solutions of the linearized problem in order, for instance, to estimate the size of the attractor in the usual energy space. Surprisingly enough, our estimates provide bounds on the attractor which in some relevant cases are optimal up to at most a $\sqrt{2}$ factor. At the present time we do not know if an optimal bound can be achieved.

The plan of the paper is the following: Section 1 contains the statement of all major results. Sections 2,3, 4 and 5 are devoted to the proofs. In Section 6 we give examples of application to the size of attractors of some nonlinear wave equations in a bounded domain. The results are shown to be almost optimal. 


\section{1- Main results.}

We start with a non-optimal result which is applicable in a rather broad framework

Theorem 1.1. Let $\beta(t): \mathbb{R} \rightarrow C\left(V, V^{\prime}\right)$ be a measurable family of continuous operators, $f \in L^{\infty}(\mathbb{R}, H)$ and assume for some constants $C>0, \quad c>0$

$$
\begin{gathered}
\forall t \in \mathbb{R}, \forall v \in V, \quad\langle\beta(t) v, v\rangle \geq c|v|^{2} \\
\forall t \in \mathbb{R}, \forall v \in V, \quad\|\beta(t) v\|_{*}^{2} \leq C\langle\beta(t) v, v\rangle
\end{gathered}
$$

Then if $u \in C^{1}(\mathbb{R}, V) \cap W_{l o c}^{2,1}(\mathbb{R}, H)$ is a bounded solution of

$$
u^{\prime \prime}+A u+\beta(t) u^{\prime}=f(t)
$$

in the sense that

$$
u \in L^{\infty}(\mathbb{R}, V), \quad u^{\prime} \in L^{\infty}(\mathbb{R}, H)
$$

We have the following estimates

$$
\begin{gathered}
\forall t \in \mathbb{R}, \quad\left\{\left|u^{\prime}(t)\right|^{2}+\|u(t)\|^{2}\right\}^{1 / 2} \leq \frac{3}{\sqrt{2}}\left(\frac{4}{c}+C\right)\|f\|_{L^{\infty}(R, H)} \\
\forall t \in \mathbb{R}, \quad \sup \left\{\left|u^{\prime}(t)\right|,\|u(t)\|\right\} \leq \sqrt{3}\left(\frac{4}{c}+C\right)\|f\|_{L^{\infty}(R, H)}
\end{gathered}
$$

Corollary 1.2. Assume that $\beta \in L^{\infty}(\mathbb{R}, \mathcal{L}(H))$ satisfies (1.1) and

$$
\forall v \in H,|\beta(t) v| \leq \Gamma|v|
$$

Then (1.4)-(1.5) are valid for any solution $u \in C_{b}^{0}(\mathbb{R}, V) \cap C_{b}^{1}(\mathbb{R}, H) \cap W^{2, \infty}\left(\mathbb{R}, V^{\prime}\right)$ with $C=\frac{\Gamma^{2}}{c \lambda_{1}}$

Corollary 1.3. In the special case

$$
\forall t \in \mathbb{R}, \forall v \in H, \quad \beta(t) v=c v
$$

we obtain

$$
\begin{gathered}
\forall t \in \mathbb{R}, \quad\left\{\left|u^{\prime}(t)\right|^{2}+\|u(t)\|^{2}\right\}^{1 / 2} \leq \frac{3}{\sqrt{2}}\left(\frac{4}{c}+\frac{c}{\lambda_{1}}\right)\|f\|_{L^{\infty}(R, H)} \\
\forall t \in \mathbb{R}, \quad \sup \left\{\left|u^{\prime}(t)\right|,\|u(t)\|\right\} \leq \sqrt{3}\left(\frac{4}{c}+\frac{c}{\lambda_{1}}\right)\|f\|_{L^{\infty}(R, H)}
\end{gathered}
$$

valid for any solution $u \in C_{b}^{0}(\mathbb{R}, V) \cap C_{b}^{1}(\mathbb{R}, H) \cap W^{2, \infty}\left(\mathbb{R}, V^{\prime}\right)$.

Corollary 1.3 is not optimal. In fact a better result can be obtained for equation (1) under suitable hypotheses on $c$ and $A$. 
We start with a result when $c$ is small (weak dissipation)

Theorem 1.4. Assume $c \leq 2 \sqrt{\lambda_{1}}$ or equivalently

$$
\lambda_{1} \geq \frac{c^{2}}{4}
$$

Then the bounded solution $u$ of (1) satisfies the stronger estimates

$$
\begin{gathered}
\forall t \in \mathbb{R}, \quad\|u(t)\| \leq \sqrt{\frac{4}{c^{2}}+\frac{1}{\lambda_{1}}} \quad\|f\|_{L^{\infty}(R, H)} \leq \frac{2 \sqrt{2}}{c}\|f\|_{L^{\infty}(R, H)} \\
\forall t \in \mathbb{R}, \quad\left|u^{\prime}(t)\right| \leq \frac{4}{c}\|f\|_{L^{\infty}(R, H)}
\end{gathered}
$$

The next intermediate result is only valid for $A$ bounded

Theorem 1.5. Let $A \in L(H)$ and assume

$$
c \geq 2\left\|A^{1 / 2}\right\|=2\|A\|^{1 / 2}
$$

Then the bounded solution $u$ of (1) satisfies the estimate

$$
\forall t \in \mathbb{R},\|u(t)\|=\left|A^{1 / 2} u(t)\right| \leq \sqrt{\frac{2}{\lambda_{1}}}\|f\|_{\infty}
$$

Finally the following result for large damping complements Theorem 1.4.

Theorem 1.6. Assuming $c>2 \sqrt{\lambda_{1}}$ or equivalently

$$
\lambda_{1}<\frac{c^{2}}{4}
$$

the bounded solution $u$ of (1) satisfies the estimate

$$
\forall t \in \mathbb{R}, \quad\|u(t)\| \leq \sqrt{\frac{8}{c^{2}}+\frac{2}{\lambda_{1}}}\|f\|_{L^{\infty}(R, H)} \leq \frac{2}{\sqrt{\lambda_{1}}}\|f\|_{L^{\infty}(R, H)}
$$

Remark 1.7. It is not difficult to check that both Theorems 1.4 and 1.6 improve the result of Corollary 1.3 by a factor at least equal to $\sqrt{12}$. However the two estimates of Theorem 1.4 and Theorem 1.6 do not match for $\lambda_{1}=\frac{c^{2}}{4}$. This suggests that these results are not optimal yet. 


\section{2-Proof of Theorem 1.1.}

First we notice that the hypotheses (1.2)-(1.3) imply

$$
c \leq \lambda_{1} C
$$

Indeed for any $v \in V, \quad v \neq 0$ we have $\|\beta(t) v\|_{*} \leq C\|v\|$ and then

$$
c|v|^{2} \leq\langle\beta(t) v, v\rangle \leq\|\beta(t) v\|_{*}\|v\| \leq C\|v\|^{2} \Longrightarrow \frac{c}{C} \leq \frac{\|v\|^{2}}{|v|^{2}}
$$

In other words

$$
\frac{c}{C} \leq \inf _{v \in V-0} \frac{\|v\|^{2}}{|v|^{2}}=\lambda_{1}
$$

Next we introduce

$$
\Phi(t)=\left|u^{\prime}(t)\right|^{2}+\|u(t)\|^{2}+\delta\left(u(t), u^{\prime}(t)\right)
$$

$\Phi$ is absolutely continuous on $\mathbb{R}$ and a.e. in t, dropping for simplicity the t-dependence we have

$$
\begin{gathered}
\Phi^{\prime}=\left\langle u^{\prime \prime}+A u, 2 u^{\prime}\right\rangle+\delta\left|u^{\prime}\right|^{2}+\delta\left\langle u^{\prime \prime}, u\right\rangle \\
=-2\left\langle\beta(t) u^{\prime}, u^{\prime}\right\rangle+\delta\left|u^{\prime}\right|^{2}+\delta\left\langle f-A u-\beta(t) u^{\prime}, u\right\rangle+2\left(f, u^{\prime}\right) \\
\Phi^{\prime} \leq-2\left\langle\beta(t) u^{\prime}, u^{\prime}\right\rangle+\delta\left|u^{\prime}\right|^{2}+\sqrt{C} \delta\|u\|\left\langle\beta(t) u^{\prime}, u^{\prime}\right\rangle^{1 / 2}-\delta\|u\|^{2}+\left(f, 2 u^{\prime}+\delta u\right)
\end{gathered}
$$

We set

$$
v=\frac{1}{\sqrt{c}}\left\langle\beta(t) u^{\prime}, u^{\prime}\right\rangle^{1 / 2} \geq\left|u^{\prime}\right|, \quad w=\|u\|
$$

and

$$
P(v, w)=(2 c-\delta) v^{2}-\delta \sqrt{C c} v w+\delta w^{2}
$$

In order for the quadratic form $P$ to be positive definite it is sufficient to have

$$
\delta<2 c, \quad \Delta=C c \delta^{2}-4 \delta(2 c-\delta)<0
$$

The second condition reads $8 c>\delta(C c+4)$ and is obviously satisfied for the choice

$$
\delta=\frac{4 c}{C c+4} \leq c
$$

We have obviously

$$
\delta=\frac{4 c}{C c+4} \leq \frac{4 \sqrt{\lambda}_{1} \sqrt{C c}}{C c+4} \leq \sqrt{\lambda}_{1}
$$


An obvious calculation shows that whenever $\alpha>0$ and $\Delta^{\prime}=\beta^{2}-\alpha \gamma<0$ we have

$$
\alpha v^{2}-2 \beta v w+\gamma w^{2} \geq\left[\frac{\alpha+\gamma}{2}-\sqrt{\beta^{2}+\left(\frac{\alpha-\gamma}{2}\right)^{2}}\right]\left(v^{2}+w^{2}\right)
$$

For the quadratic form $P$ we have

$$
\frac{\alpha+\gamma}{2}=c ; \quad \beta=\frac{1}{2} \sqrt{C c} \delta ; \quad \alpha-\gamma=2(c-\delta)
$$

and

$$
c-\delta=\frac{C c^{2}}{C c+4} ; \quad \beta^{2}=\frac{C c \delta^{2}}{4}=\frac{4 C c^{3}}{(C c+4)^{2}}
$$

hence

$$
\beta^{2}+\left(\frac{\alpha-\gamma}{2}\right)^{2}=\frac{C^{2} c^{4}+4 C c^{3}}{(C c+4)^{2}}=\frac{C c^{3}}{C c+4}
$$

So we find

$$
P(v, w) \geq\left[c-\frac{c \sqrt{C c}}{\sqrt{C c+4}}\right]\left(v^{2}+w^{2}\right)
$$

Now

$$
1-\frac{\sqrt{C c}}{\sqrt{C c+4}}=\frac{\sqrt{C c+4}-\sqrt{C c}}{\sqrt{C c+4}}=\frac{4}{\sqrt{C c+4}(\sqrt{C c}+\sqrt{C c+4})} \geq \frac{2}{C c+4}
$$

and finally

$$
P(v, w) \geq \frac{2 c}{C c+4}\left(v^{2}+w^{2}\right)
$$

From (2.3) we deduce

$$
\Phi^{\prime} \leq-\frac{2 c}{C c+4}\left(\left|u^{\prime}\right|^{2}+\|u\|^{2}\right)+\left(f, 2 u^{\prime}+\delta u\right)
$$

Since by $(2.5)$

$$
\left|2 u^{\prime}+\delta u\right|^{2}=4\left|u^{\prime}\right|^{2}+4 \delta\left(u, u^{\prime}\right)+\delta^{2}|u|^{2} \leq 4\left|u^{\prime}\right|^{2}+4 \delta\left(u, u^{\prime}\right)+\|u\|^{2} \leq 4 \Phi
$$

we deduce from (2.6)

$$
\Phi^{\prime} \leq-\frac{2 c}{C c+4}\left(\left|u^{\prime}\right|^{2}+\|u\|^{2}\right)+2|f| \sqrt{\Phi}
$$

On the other hand, let

$$
E=E(t):=\left|u^{\prime}(t)\right|^{2}+\|u(t)\|^{2}
$$


Since $\Phi=E+\delta\left(u, u^{\prime}\right)$ and

$$
\left|\delta\left(u, u^{\prime}\right)\right| \leq \sqrt{\lambda_{1}}|u|\left|u^{\prime}\right| \leq\|u\|\left|u^{\prime}\right| \leq \frac{1}{2} E
$$

we have

$$
\frac{1}{2} E \leq \Phi \leq \frac{3}{2} E
$$

From (2.7) and (2.8) we deduce

$$
\Phi^{\prime} \leq-\frac{4 c}{3(C c+4)} \Phi+2|f| \sqrt{\Phi}
$$

and combining with

$$
2|f| \sqrt{\Phi} \leq \frac{2 c}{3(C c+4)} \Phi+\frac{3(C c+4)}{2 c}|f|^{2}
$$

we infer, a.e in $t$

$$
\Phi^{\prime} \leq-\frac{2 c}{3(C c+4)} \Phi+\frac{3(C c+4)}{2 c}|f|^{2}
$$

From (2.9) we deduce immediately, using boundedness of $\Phi$ on $\mathbb{R}$

$$
\forall t \in \mathbb{R}, \quad \Phi(t) \leq\left[\frac{3(C c+4)}{2 c}\right]^{2}\|f\|_{L^{\infty}(R, H)}^{2}
$$

As a first consequence we find

$$
\sqrt{E} \leq \sqrt{2 \Phi} \leq \frac{3(C c+4)}{c \sqrt{2}}\|f\|_{L^{\infty}(R, H)}
$$

hence (1.4) In addition since

$$
\Phi \geq\left|u^{\prime}(t)\right|^{2}+\|u(t)\|^{2}-\left|u^{\prime}(t)\right|\|u(t)\|
$$

we deduce easily

$$
\Phi \geq \frac{3}{4} \sup \left\{\left|u^{\prime}(t)\right|^{2},\|u(t)\|^{2}\right\}
$$

and (1.5) follows immediately from (2.10)-(2.11).

Proof of Corollary 1.2. We have

$$
\forall t \in \mathbb{R}, \forall v \in V, \quad\|\beta(t) v\|_{*}^{2} \leq \frac{1}{\lambda_{1}}|\beta(t) v|^{2} \leq \frac{\Gamma^{2}}{\lambda_{1}}|v|^{2} \leq \frac{\Gamma^{2}}{c \lambda_{1}}\langle\beta(t) v, v\rangle
$$


hence (1.2) is satisfied with $C=\frac{\Gamma^{2}}{c \lambda_{1}}$. Then Corollary 1.2 follows at once from Theorem1.1 combined with a standard density argument.

Proof of Corollary 1.3. When $\beta(t) v \equiv c v$ we deduce

$$
\forall t \in \mathbb{R}, \forall v \in V, \quad\langle\beta(t) v, v\rangle=c|v|^{2}
$$

and the hypothesis of Corollary 1.2 is satisfied with $\Gamma=c$. Then Corollary 1.3 follows at once from Corollary 1.2.

\section{3- The case of a small damping.}

This section is devoted to the proof of Theorem 1.4. Under hypothesis (1.8) we can use the specific, well-adapted, energy functional already used in $[6,7]$ :

$$
\Phi(t)=\left|u^{\prime}(t)\right|^{2}+\|u(t)\|^{2}+c\left(u(t), u^{\prime}(t)\right)
$$

Here we have

$$
\begin{gathered}
\Phi^{\prime}=\left\langle u^{\prime \prime}+A u, 2 u^{\prime}\right\rangle+c\left|u^{\prime}\right|^{2}+c\left\langle u^{\prime \prime}, u\right\rangle=-c\left|u^{\prime}\right|^{2}+c\left\langle f-A u-c u^{\prime}, u\right\rangle+2\left(f, u^{\prime}\right) \\
\Phi^{\prime}=-c \Phi+\left(f, 2 u^{\prime}+c u\right)
\end{gathered}
$$

On the other hand

$$
\left|2 u^{\prime}+c u\right|^{2}=4\left|u^{\prime}\right|^{2}+4\left(u, u^{\prime}\right)+c^{2}|u|^{2} \leq 4\left|u^{\prime}\right|^{2}+4 c\left(u, u^{\prime}\right)+4\|u\|^{2} \leq 4 \Phi
$$

hence, using

$$
\left(f, 2 u^{\prime}+c u\right) \leq \frac{2}{c}|f|^{2}+\frac{c}{8}\left|2 u^{\prime}+c u\right|^{2} \leq \frac{2}{c}|f|^{2}+\frac{c}{2} \Phi
$$

we deduce from (2.13) the inequality

$$
\Phi^{\prime} \leq-\frac{c}{2} \Phi+\frac{2}{c}|f|^{2}
$$

In particular, since $\Phi$ is bounded we find

$$
\forall t \in \mathbb{R}, \quad \Phi(t) \leq \frac{4}{c^{2}}\|f\|_{\infty}^{2}
$$

which means

$$
\forall t \in \mathbb{R}, \quad\left|u^{\prime}(t)\right|^{2}+\|u(t)\|^{2}+c\left(u(t), u^{\prime}(t)\right) \leq \frac{4}{c^{2}}\|f\|_{\infty}^{2}
$$


In particular

$$
\forall t \in \mathbb{R}, \quad \lambda_{1}|u(t)|^{2}+c\left(u(t), u^{\prime}(t)\right) \leq \frac{4}{c^{2}}\|f\|_{\infty}^{2}
$$

and this means

$$
\frac{c}{2}\left(|u(t)|^{2}\right)^{\prime}+\lambda_{1}|u(t)|^{2} \leq \frac{4}{c^{2}}\|f\|_{\infty}^{2}
$$

Along with boundedness of $u(t)$ in $H$ on $\mathbb{R}$ this implies

$$
\forall t \in \mathbb{R}, \quad|u(t)|^{2} \leq \frac{4}{c^{2} \lambda_{1}}\|f\|_{\infty}^{2}
$$

Finally from (2.15) we deduce

$$
\begin{aligned}
\forall t \in \mathbb{R}, \quad\|u(t)\|^{2} \leq \frac{4}{c^{2}}\|f\|_{\infty}^{2} & -\left|u^{\prime}(t)\right|^{2}-c\left(u(t), u^{\prime}(t)\right) \leq \frac{4}{c^{2}}\|f\|_{\infty}^{2}+\frac{c^{2}}{4}|u(t)|^{2} \\
& \leq\left(\frac{4}{c^{2}}+\frac{1}{\lambda_{1}}\right)\|f\|_{\infty}^{2}
\end{aligned}
$$

hence (1.9) is established. To check (1.10) we start from (2.15)-(2.16) which provide

$$
\forall t \in \mathbb{R}, \quad\left|u^{\prime}(t)\right|^{2}+2 \lambda_{1}|u(t)|^{2}+c\left(u(t), u^{\prime}(t)\right) \leq \frac{8}{c^{2}}\|f\|_{\infty}^{2}
$$

in particular

$$
\forall t \in \mathbb{R}, \quad\left|u^{\prime}(t)\right|^{2}+\frac{c^{2}}{2}|u(t)|^{2}+c\left(u(t), u^{\prime}(t)\right) \leq \frac{8}{c^{2}}\|f\|_{\infty}^{2}
$$

hence

$$
\forall t \in \mathbb{R}, \quad\left|u^{\prime}(t)\right|^{2} \leq \frac{8}{c^{2}}\|f\|_{\infty}^{2}-\frac{c^{2}}{2}|u(t)|^{2}-c\left(u(t), u^{\prime}(t)\right) \leq \frac{8}{c^{2}}\|f\|_{\infty}^{2}+\frac{1}{2}\left|u^{\prime}(t)\right|^{2}
$$

from which (1.10) follows at once.

Remark 3.1. Compared with the scalar case we lose here a factor $\sqrt{2}$. This extra factor disappears using the same proof only when $A=\lambda_{1} I$, a special situation which generalizes the scalar case, in which case the result is optimal for $c=2 \sqrt{\lambda_{1}}$. We do not know whether it is possible to do any better in general, even when $H=\mathbb{R}^{2}$.

\section{4- Bounded operator and large damping}

This section is devoted to the proof of Theorem 1.5. We shall use the following simple lemma. 
Lemma 4.1 Let $B \in L(H)$ satisfy $B \geq \eta I$ with $\eta>0$. Then for each $f \in$ $L^{\infty}(\mathbb{R}, H)$ there exists one and only one solution $u$ bounded on $\mathbb{R}$ with values in $H$ of

$$
u^{\prime}+B u=f
$$

and we have

$$
\forall t \in \mathbb{R}, \quad\left|B^{1 / 2} u(t)\right| \leq \frac{1}{\sqrt{\eta}}\|f\|_{L^{\infty}(R, H)}
$$

Proof. Let $u$ be a solution of (4.1) on some open interval $J \subset \mathbb{R}$ and introduce

$$
\|u\|=\left|B^{1 / 2} u\right|
$$

We have for almost all $t \in J$

$$
\frac{d}{d t}\|u\|^{2}+2(B u, B u)=2(f, B u) \leq|f|^{2}+(B u, B u)
$$

hence

$$
\frac{d}{d t}\|u\|^{2}+\eta\|u\|^{2} \leq \frac{d}{d t}\|u\|^{2}+|B u|^{2} \leq|f|^{2}
$$

In the special case $f=0$, this shows that the semi-group $\exp (-t B)$ is exponentially decaying, implying existence and uniqueness of the bounded solution $u$. Now applying the above inequality to the bounded solution $u$ on $J=\mathbb{R}$ we find by a standard procedure

$$
\eta\left(\sup _{R}\|u\|\right)^{2}=\eta \sup _{R}\|u\|^{2} \leq\|f\|_{L^{\infty}(R, H)}^{2}
$$

from which (4.2) follows immediately.

Proof of Theorem 1.5. As a consequence of (1.11) the operator

$$
D=c^{2} I-4 A
$$

satisfies $D=D^{*} \geq 0$ and we can define

$$
B=\frac{c I+D^{1 / 2}}{2}, \quad C=\frac{c I-D^{1 / 2}}{2}
$$

It is readily verified that

$$
B+C=c I ; \quad B C=C B=A
$$


In addition since $c^{2} I-4 A \geq\left(c^{2}-4\|A\|\right) I$ we deduce

$$
B \geq \frac{c+\sqrt{c^{2}-4\|A\|}}{2} I
$$

and since $c^{2} I-4 A \leq\left(c^{2}-4 \lambda_{1}\right) I$ we have

$$
C \geq \frac{c-\sqrt{c^{2}-4 \lambda_{1}}}{2} I
$$

As a consequence of Lemma 4.1, for each $f \in L^{\infty}(\mathbb{R}, H)$ there is a unique bounded solution $v$ of

$$
v^{\prime}+B v=f
$$

Since $v$ is bounded there is a unique bounded solution $u$ of

$$
u^{\prime}+C u=v
$$

It is clear that

$$
u, v \in W^{1, \infty}(\mathbb{R}, H)
$$

Then $u^{\prime}=v-C u \in W^{1, \infty}(\mathbb{R}, H)$ and we have

$$
u^{\prime \prime}=v^{\prime}-C u^{\prime}=f-B v-C u^{\prime}=f-C u^{\prime}-B\left(u^{\prime}+C u\right)=f-c u^{\prime}-A u
$$

Finally, $u$ is the bounded solution of

$$
u^{\prime \prime}+c u^{\prime}+A u=f
$$

on the other hand by Lemma 4.1 we have, denoting by $\|u\|_{\infty}$ the norm of $u$ in $L^{\infty}(\mathbb{R}, H)$

$$
\left\|B^{1 / 2} v\right\|_{\infty} \leq \frac{1}{\sqrt{\lambda_{1}(B)}}\|f\|_{\infty}
$$

Letting

$$
w=B^{1 / 2} v
$$

we then have

$$
\left(B^{1 / 2} u\right)^{\prime}+C B^{1 / 2} u=w
$$

and by Lemma 4.1 applied to $C$ we find

$$
\left\|A^{1 / 2} u\right\|_{\infty}=\left\|C^{1 / 2} B^{1 / 2} u\right\|_{\infty} \leq \frac{1}{\sqrt{\lambda_{1}(C)}}\|w\|_{\infty} \leq \frac{1}{\sqrt{\lambda_{1}(B) \lambda_{1}(C)}}\|f\|_{\infty}
$$


which means

$$
\forall t \in \mathbb{R}, \quad\|u(t)\| \leq \frac{1}{\sqrt{\lambda_{1}(B) \lambda_{1}(C)}}\|f\|_{\infty}
$$

Now we have

$$
\lambda_{1}(B) \geq \frac{c+\sqrt{c^{2}-4\|A\|}}{2} ; \quad \lambda_{1}(C) \geq \frac{c-\sqrt{c^{2}-4 \lambda_{1}}}{2}
$$

Because

$$
c-\sqrt{c^{2}-4 \lambda_{1}}=\frac{4 \lambda_{1}}{c+\sqrt{c^{2}-4 \lambda_{1}}}
$$

and since

$$
c \leq c+\sqrt{c^{2}-4\|A\|} \leq c+\sqrt{c^{2}-4 \lambda_{1}} \leq 2 c
$$

we find

$$
\lambda_{1}(B) \lambda_{1}(C) \geq \lambda_{1} \frac{c+\sqrt{c^{2}-4\|A\|}}{c+\sqrt{c^{2}-4 \lambda_{1}}} \geq \frac{\lambda_{1}}{2}
$$

and finally

$$
\forall t \in \mathbb{R}, \quad\|u(t)\| \leq \sqrt{\frac{2}{\lambda_{1}}}\|f\|_{\infty}
$$

and this concludes the proof of (1.12).

Remark 4.2. a) The proof of Theorem 1.5 may look somewhat strange but it is in fact a natural generalization of the proof of Theorem 2.1, i) from [7] which in turn is related to the main principle of $[1,9]$ in which an order preserving property was used. The idea is just to decompose the second order operator with boundedness condition at infinity as a product of two first order operators which are easier to handle. The decomposition turns out to be real precisely under condition (1.11). When (1.11) is not satisfied, the decomposition gives a weak estimate even in the scalar case, this explains why we use a different method for the proof of Theorem 1.4.

b) Compared with the scalar case we lose here a factor $\sqrt{2}$. Actually when $A=\lambda_{1} I$ the $\sqrt{2}$ factor disappears and we recover the scalar case. We do not know whether it is possible to avoid the extra $\sqrt{2}$ factor in general, even when $H=\mathbb{R}^{2}$. At least the $\sqrt{2}$ factor can always be replaced by the smaller factor

$$
c(A)=\sqrt{\frac{c+\sqrt{c^{2}-4 \lambda_{1}}}{c+\sqrt{c^{2}-4\|A\|}}}
$$




\section{5- Proof of Theorem 1.6.}

We now prove Theorem 1.6. As a consequence of [11], Theorem VIII.4 p. 260, we may assume, up to an isometric isomorphism, that

$$
H=L^{2}(E, d \mu)
$$

and

$$
\begin{gathered}
D(A)=L^{2}\left(E,\left(1+a^{2}\right) d \mu\right) \\
\forall u \in D(A), \quad(A u)(x)=a(x) u(x), \mu-a . e
\end{gathered}
$$

Now let

$$
\begin{aligned}
& H_{+}=\left\{u \in H, \quad u(x)=0 \quad \mu-\text { a.e. on }\left\{a(x) \geq \frac{c^{2}}{4}\right\}\right\} \\
& H_{-}=\left\{u \in H, \quad u(x)=0 \quad \mu \text {-a.e. on }\left\{a(x)<\frac{c^{2}}{4}\right\}\right\}
\end{aligned}
$$

Let $P$ and $Q=I-P$ be the orthogonal projectors on $H_{+}$and $H_{-}$respectively. Given $f \in L^{\infty}(\mathbb{R}, H)$ we remark that the bounded solution of (1) can be written

$$
u=P u+Q u
$$

where $(P u, Q u)$ lie in $H_{+}$and $H_{-}$respectively and are the bounded solutions associated respectively to $(P f, Q f)$. Applying Theorem 1.4 to $(P u, P f)$ in $H_{+}$we find

$$
\forall t \in \mathbb{R}, \quad\|P u(t)\| \leq \frac{2 \sqrt{2}}{c}\|P f\|_{L^{\infty}(R, H)}
$$

and by applying Theorem 1.5 to $(Q u, Q f)$ in $H_{-}$we find

$$
\forall t \in \mathbb{R},\|Q u(t)\| \leq \sqrt{\frac{2}{\lambda_{1}}}\|Q f\|_{L^{\infty}(R, H)}
$$

Then

$$
\forall t \in \mathbb{R}, \quad\|u(t)\|^{2} \leq\|P u(t)\|^{2}+\|Q u(t)\|^{2} \leq\left(\frac{8}{c^{2}}+\frac{2}{\lambda_{1}}\right)\|f\|_{L^{\infty}(R, H)}^{2}
$$

and (1.13) follows easily. 


\section{6- Applications.}

Let $(\Omega, \mu)$ be a finitely measured space and $A$ a positive definite self-adjoint linear operator as in the introduction on $H=L^{2}(\Omega, d \mu)$.

\subsection{Local dissipations associated to bounded potentials.}

Theorem 1.1 and its corollaries can be applied to estimate the bounded solution $u \in C_{b}(\mathbb{R}, V) \cap C_{b}^{1}(\mathbb{R}, H) \cap W^{2, \infty}\left(\mathbb{R}, V^{\prime}\right)$ of the equation

$$
u^{\prime \prime}+A u+c(t, x) u^{\prime}=f(t, x)
$$

when $c \in L^{\infty}(\mathbb{R} \times \Omega)$ is such that

$$
0<\gamma \leq c(t, x) \leq \Gamma, \quad \text { a.e on } \mathbb{R} \times \Omega
$$

as a consequence of Corollary 1.2 we have

$$
\begin{gathered}
\forall t \in \mathbb{R}, \quad\left\{\left|u^{\prime}(t)\right|^{2}+\|u(t)\|^{2}\right\}^{1 / 2} \leq \frac{3}{\sqrt{2}}\left(\frac{4}{\gamma}+\frac{\Gamma^{2}}{\gamma \lambda_{1}}\right)\|f\|_{L^{\infty}(R, H)} \\
\forall t \in \mathbb{R}, \quad \sup \left\{\left|u^{\prime}(t)\right|,\|u(t)\|\right\} \leq \sqrt{3}\left(\frac{4}{\gamma}+\frac{\Gamma^{2}}{\gamma \lambda_{1}}\right)\|f\|_{L^{\infty}(R, H)}
\end{gathered}
$$

Similar estimates can be derived when $\Omega$ is a bounded domain in $\mathbb{R}^{N}$ and for instance

$$
V=H_{0}^{1}(\Omega) ; \quad A=-\Delta
$$

assuming

$$
c(t, x) \geq \gamma>0, \quad c \in L^{\infty}\left(\mathbb{R}, L^{p}(\Omega)\right)
$$

with $p \geq 2$ if $N=1, p>2$ if $N=2, p \geq N$ if $N \geq 3$. We leave the estimates to the reader as an easy excercise.

\subsection{More general damping operators.}

It is possible to use Theorem 1.1 to study such problems as

$$
u^{\prime \prime}-\Delta u+b(x) u+c(t, x) u^{\prime}-\gamma(t) \Delta u^{\prime}=f(t, x)
$$

or

$$
u^{\prime \prime}+\Delta^{2} u+b(x) u+c(t, x) u^{\prime}-\gamma(t) \Delta u^{\prime}+\sigma(t) \Delta^{2} u^{\prime}=f(t, x)
$$


with various boundary conditions. But these examples deserve a detailed analysis which would certainly provide sharper results than a simple use of Theorem 1.1, especially when $c$ is a constant. The estimates provided by Theorem 1.1 are a bit tedious in general and are left again as an exercise.

\subsection{Bound on attractors of sine-telegraph equations.}

Assuming $\mu(\Omega)<\infty$, let us consider a bounded function $F: \mathbb{R} \rightarrow[-a,+a]$ and let $u \in C_{b}(\mathbb{R}, V) \cap C_{b}^{1}(\mathbb{R}, H) \cap W^{2, \infty}\left(\mathbb{R}, V^{\prime}\right)$ be a solution of

$$
u^{\prime \prime}+c u^{\prime}+A u=F(u)
$$

Then if $c \geq 2 \sqrt{\lambda_{1}}$ we have

$$
\forall t \in \mathbb{R}, \quad\|u(t)\| \leq \frac{2 a}{\sqrt{\lambda}_{1}} \mu(\Omega)^{1 / 2}
$$

and if $c \leq 2 \sqrt{\lambda_{1}}$ we have

$$
\forall t \in \mathbb{R}, \quad\|u(t)\| \leq \frac{2 a \sqrt{2}}{c} \mu(\Omega)^{1 / 2}
$$

For instance, let $\Omega$ be a bounded open domain in $\mathbb{R}^{N}$ and $b \geq 0, c>0$ We consider the problem

$$
u^{\prime \prime}-\Delta u+b u+c u^{\prime}=a \sin u
$$

with one of the boundary conditions

$$
u=0 \quad \text { on } \partial \Omega
$$

or

$$
\frac{\partial u}{\partial n}=0 \quad \text { on } \partial \Omega
$$

It is well known, c.f. eg [6] that problems (6.10)-(6.11) and (6.10)-(6.12) have a compact attractor $\mathcal{A}$ under the condition $b>0$ in the second case. Our results allow to obtain an upper bound of the size of the $u$-projection of $\mathcal{A}$ since the attractor is just the union of the ranges of bounded solutions. In the case of problem (6.10)-(6.11) we have

$$
\mathcal{A} \subset\left\{(u, v) \in H_{0}^{1}(\Omega) \times L^{2}(\Omega), \quad\left\{\int_{\Omega}\|\nabla u\|^{2} d x\right\}^{1 / 2} \leq \frac{2 a \sqrt{2}}{c}|\Omega|^{1 / 2}\right\}
$$


whenever

$$
c \leq 2 \sqrt{\lambda_{1}(\Omega)+b}
$$

and for problem (6.10)-(6.12) we have

$$
\mathcal{A} \subset\left\{(u, v) \in H_{0}^{1}(\Omega) \times L^{2}(\Omega), \quad\left\{\int_{\Omega}\left(\|\nabla u\|^{2}+b u^{2}\right) d x\right\}^{1 / 2} \leq \frac{2 a \sqrt{2}}{c}|\Omega|^{1 / 2}\right\}
$$

whenever

$$
c \leq 2 \sqrt{b}
$$

These results are, surprisingly enough, close to optimality. For instance in the second case, for specific values of the parameters the problem has stationary solutions, constant in time and space. These are the solutions of

$$
u \in \mathbb{R}, \quad b u=a \sin u
$$

The estimate (6.14) here means that any solution $u$ of (6.15) satisfies

$$
|u| \leq \frac{2 a \sqrt{2}}{c \sqrt{b}}
$$

let us consider the limiting case

$$
c=2 \sqrt{b}
$$

Then the above inequality becomes

$$
|u| \leq \frac{a \sqrt{2}}{b}
$$

Now let $k \in \mathbb{N}, k>1$ and let

$$
\varepsilon_{k}=\frac{1}{(2 k+1) \pi}
$$

We have

$$
\sin \left(\frac{\pi}{2}+2 k \pi\right)-\varepsilon_{k}\left(\frac{\pi}{2}+2 k \pi\right)>0>\sin ((2 k+1) \pi)-\varepsilon_{k}(2 k+1) \pi=-1
$$

and it follows :

$$
\exists u_{k} \in\left[\frac{\pi}{2}+2 k \pi,(2 k+1) \pi\right], \quad \sin u_{k}=\varepsilon_{k} u_{k}
$$

Now select any pair $(a, b)$ of positive numbers with

$$
a=(2 k+1) \pi b
$$


our inequality shows

$$
u_{k} \leq(2 k+1) \pi \sqrt{2}
$$

while the actual $u_{k}$ is such that

$$
u_{k} \geq \frac{\pi}{2}+2 k \pi
$$

For $k$ large we are off by a factor $\sqrt{2}$. So that for any $\Omega$ our estimate is sharp up to a $\sqrt{2}$ multiplicative constant. And of course in general the attractor may contain non stationary solutions.

Remark. It would of course be possible to estimate also the size in $L^{2}(\Omega)$ of the $u^{\prime}$-projection of the attractor. But in this case it is more difficult to study the optimality, so we did not give an estimate for which we have no optimality result.

\section{References}

1. J.M. Alonso, J. Mawhin \& R. Ortega, Bounded solutions of second order semilinear evolution equations and applications to the telegraph equation, J. Math. Pures Appl. 78 (1999), 49-63.

2. M.L. Cartwright \& J.E. Littlewood, On nonlinear differential equations of the second order, Annals of Math 48 (1947), 472-494.

3. S.N. Chow, J.K. Hale \& J. Mallet-Paret, An example of bifurcation to homoclinic orbits, J.D.E. 37 (1980), 351-373.

4. J.K. Hale \& P.Z. Taboas, Interaction of damping and forcing in a second order evolution equation, Nonlinear analysis, T.M.A. 2, 1 (1978), 77-84.

5. A. Haraux, Nonlinear evolution equations: Global behavior of solutions, Lecture Notes in Math. 841, Springer (1981)

6. A. Haraux, Systèmes dynamiques dissipatifs et applications, R.M.A.17, P.G. Ciarlet et J.L. Lions (eds.), Masson, Paris, 1991.

7. A. Haraux, On the double well Duffing equation with a small bounded forcing term, to appear in Rend. Accad. Nazionale delle Scienze detta dei XL, Memorie di Matematica (2006).

8. W. S. Loud, Boundedness and convergence of solutions of $x^{\prime \prime}+c x^{\prime}+g(x)=e(t)$, Mem. Amer. Math. Soc., 31, 1959, 1-57. 
9. J. Mawhin, R. Ortega \& A.M. Robles-Perez, A maximun principle for bounded solutions of the telegraph equations and applications to nonlinear forcings, J. Math. Anal. Appl. 251 (2000), 695-709.

10. F. C. Moon \& P. J. Holmes, A magnetoelastic strange attractor, Journal of Sound and Vibration 65, 2 (1979), 275-296.

11. M. Reed \& B. Simon, Methods of Modern Mathematical Physics I: Functional Analysis, 2nd Edition, Academic press, New-York (1980). 